\title{
Ten-year all-cause mortality in hospitalized non-surgical patients based on nutritional status screening
}

\author{
Viera Kissova ${ }^{1, *}$, Jaroslav Rosenberger ${ }^{2}$, Maria Goboova $^{1}$ and Adrian Kiss ${ }^{3}$ \\ 'Department of Internal Medicine, Internal Clinic, Teaching Hospital Nitra, Spitalska 6, 94901 Nitra, Slovak \\ Republic: ${ }^{2}$ Institute of Public Health and Graduate School Kosice, Institute for Society and Health, University PJ \\ Safarik, Kosice, Slovak Republic: ${ }^{3}$ Medical Faculty, Comenius University, Bratislava, Slovak Republic
}

Submitted 11 February 2014: Final revision received 3 September 2014: Accepted 15 December 2014: First published online 5 February 2015

\begin{abstract}
Objective: Malnutrition is common in patients admitted to hospital due to acute illness and contributes to negative patient outcomes. In Slovakia there is a lack of relevant data on malnutrition in hospitalized patients, particularly based on chronic co-morbidity and survival. The aim of the present study was to explore the prevalence of malnutrition in hospitalized chronic patients, its relationship to co-morbidity and its impact on 10-year survival.

Design: Retrospective cohort study.

Setting: Nutritional status was estimated by Subjective Global Assessment (SGA), BMI and serum albumin level. Survival was assessed from the National Insurance Registry over a 10-year period. The association between nutritional status measured by SGA and 10-year survival controlling for age, gender, BMI and serum albumin was analysed using Cox regression.

Subjects: Data were taken from the medical records of 202 consecutively admitted chronic patients.

Results: Median age was 63.5 years; $55.4 \%$ were males; median BMI was $25.9 \mathrm{~kg} / \mathrm{m}^{2}$; median serum albumin level was $39.0 \mathrm{~g} / \mathrm{l}$. Based on SGA evaluation, $38.1 \%$ did not have sufficient nutritional status (SGA classification B and C). Malnutrition was more common in patients who were older $(P=0.023)$, with lower BMI $(P<0 \cdot 001)$, who had gastrointestinal $(P=0.049)$ and oncologic co-morbidity $(P=0.021)$ and lower albumin level $(P=0 \cdot 049)$. In-hospital mortality was $3 \%$, but during the following 10 years $52 \%$ died. Cox regression analysis controlling for age, gender, BMI and serum albumin showed that SGA was an independent predictor of death (hazard ratio $=1 \cdot 55 ; 95 \%$ CI $1.04,2 \cdot 32 ; P=0 \cdot 031)$.

Conclusions: SGA is a simple screening tool that can be routinely used in hospitalized Slovak medical patients to predict the risk of death. Improving patient nutrition could thus reduce mortality.
\end{abstract}

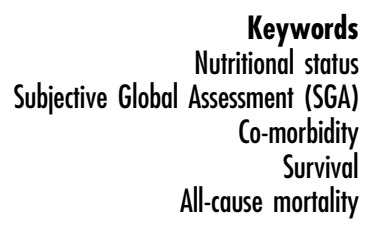

Malnutrition in the form of unintentional weight loss of more than $10 \%$ of body weight is common among acutely and chronically ill medical patients in hospitals ${ }^{(1,2)}$. The presence of malnutrition adversely affects clinical outcomes and indicates a need to target this population ${ }^{(3)}$.

Many factors alter nutritional status ${ }^{(4)}$; therefore, it is difficult to estimate the optimal approach to nutritional assessment. However, serum albumin level can be influenced by body fluid distribution, post-operative state and renal and hepatic function ${ }^{(5)}$. BMI, a simple anthropometric measure, is the most frequently used parameter of nutritional status and is associated with all-cause mortality risk in older people, mostly driven by an increased risk of cardiovascular mortality ${ }^{(6)}$.
On the other hand, Detsky et $a l .{ }^{(7)}$ introduced the Subjective Global Assessment (SGA), which provides an integration of historical (weight loss, dietary intake) and physical (loss of subcutaneous muscle mass or fat mass) data. The application of SGA in nutritional risk screening enhances the ability to predict poor clinical outcomes ${ }^{(8)}$. Thus far, SGA as a malnutrition screening tool has been a good predictor of health-related quality of life in chronic kidney disease as well ${ }^{(9)}$. BMI and SGA have been used in many studies as predictors of malnutrition prevalence, clinical outcomes and mortality in different periods of follow-up ${ }^{(8,10)}$.

Data from European countries on the prevalence of malnutrition in different clinical settings ${ }^{(11-13)}$ are well known; 
however, general data about the prevalence of malnutrition in Slovak hospitals are uncommon. Thus, we aimed to explore the prevalence of malnutrition in medical (nonsurgical) patients admitted to hospital due to acute or chronic illness, using both SGA and BMI as routine screening tools. In addition, we aimed to evaluate the correlation between serum albumin levels and both SGA and BMI as assessment tools of malnutrition. We investigated co-morbid diseases as an important factor of clinical outcome and the impact of nutritional status on the 10-year survival of these patients.

\section{Participants and methods}

This was a prospective observational cohort study. All patients hospitalized at the Internal Clinic of the Nitra Teaching Hospital from April 2003 until June 2003 were consecutively included (243 patients). Out of these patients, twenty-two did not agree to participate or could not provide informed consent and nineteen were excluded due to multi-organ failure, resulting in 202 patients included in the study.

Patient nutritional status at admission (up to $12 \mathrm{~h}$ ) was evaluated by a trained physician using the SGA protocol. SGA is an appropriate tool for assessing malnutrition ${ }^{(8,11)}$. With regard to SGA we classified patients into two groups: (i) normal nutrition (good nutritional status), SGA classification A; and (ii) malnutrition (moderate or severe), SGA classification $\mathrm{B}$ and $\mathrm{C}$.

Body weight and height were measured by an electronic scale with a separate stadiometer. BMI was calculated for every patient (weight and height in the patient's admission protocol sheet) within the first $12 \mathrm{~h}$ of the hospital stay. BMI evaluation was based on the WHO 1998 criteria with the following cut-off points: obesity, $B M I \geq 30 \cdot 0 \mathrm{~kg} / \mathrm{m}^{2}$; overweight, $\mathrm{BMI}=25.0-29.9 \mathrm{~kg} / \mathrm{m}^{2}$; normal weight, BMI $18.5-24.9 \mathrm{~kg} / \mathrm{m}^{2}$; and underweight, BMI $<18.5 \mathrm{~kg} / \mathrm{m}^{2(14)}$. Nevertheless, the use of BMI to assess nutritional status in elderly patients over 65 years old is widely debated due to body composition alterations ${ }^{(15)}$.

Blood samples were drawn from the venous line for determination of serum albumin level. Albumin was assessed by colorimetric assay with the bromocresol green method in a local laboratory as a standard part of diagnostic procedures, with references values between 39 and $42 \mathrm{~g} / 1$.

Co-morbidity data were extracted from medical and nursing investigation records. The following diseases were recorded: CVD (angina pectoris, myocardial infarction, positive coronarography, congestive heart disease (New York Heart Association Functional Classification, class III and IV), history of stroke); respiratory diseases (bronchial asthma, chronic obstructive pulmonary disorder (Global initiative for chronic Obstructive Lung Disease, since stage III), restrictive pulmonary disorders); neurological diseases (multiple sclerosis, myasthenia gravis, stroke within 3 months, polyradiculoneuritis, dementia); endocrine diseases (thyroid disorders, diabetes mellitus); nephrologic diseases (chronic kidney disease); liver diseases (cirrhosis scoring, Child/Pugh since B/B); gastrointestinal diseases (inflammatory bowel disease based on histology, acute and chronic pancreatitis, coeliac disease); systemic diseases (systemic collagen and vascular diseases such as vasculitis, rheumatoid arthritis); oncologic diseases (active disease with ongoing treatment by chemotherapy or radiotherapy, except skin malignancies); and others (intoxication, psychotic disorders).

Information about patient survival was collected from the registry of the Healthcare Surveillance Authority until June 2012. The local ethics committee of the Nitra Teaching Hospital in Slovakia agreed the research proposal.

\section{Statistics}

Descriptive statistics (frequencies, medians and interquartile ranges (IQR)) were used for the sample description. Associations between SGA and the categorical variables were evaluated using the $\chi^{2}$ test; between SGA and continuous variables using the Mann-Whitney $U$ test. Cox regression was used to explore the association between mortality and a model made up of the following variables: age, gender, BMI, albumin serum level and SGA evaluation. In the second model co-morbidity was also added (only variables significant in bivariate analysis). Kaplan-Meier curves were plotted for survival of patients with normal nutrition and malnutrition. The statistical software package IBM SPSS Statistics version 20 was used for statistical analyses.

\section{Results}

No significant differences were found between participants and non-participants regarding age and gender. The median age of patients was 63.5 (IQR 51.0-74.0) years; 112 of them were males (55.4\%); median BMI was 25.9 (IQR $22 \cdot 1-29 \cdot 0) \mathrm{kg} / \mathrm{m}^{2}$; and median serum albumin level was 39.0 (IQR 34.3-43.0) g/). Based on SGA evaluation, sixty-eight patients were malnourished (SGA classification B) and nine patients were severely malnourished (SGA classification $\mathrm{C}$ ); due to the small number in the SGA C group we merged these two categories. Detailed information on the sample divided into normal nutrition (SGA classification A) and malnutrition (SGA classification B and $\mathrm{C}$ ) is given in Table 1 ; the major reasons for hospitalization are described in Table 2.

Malnutrition was more common in older patients $(P=0.023)$, patients with lower BMI $(P<0.001)$, with gastrointestinal co-morbidity $\left(\chi^{2}=10 \cdot 7, P=0 \cdot 001\right)$, oncologic co-morbidity $\left(\chi^{2}=5 \cdot 35, P=0.021\right)$ and those with lower serum albumin $(P=0 \cdot 049)$.

The median follow-up period was 8.5 (IQR 3-8.5) years. The median period of follow-up for patients with 
Table 1 Baseline characteristics according to nutritional status among hospitalized non-surgical patients ( $n$ 202; median age 63.5 years) admitted to Nitra Teaching Hospital, Slovak Republic, April-June 2003

\begin{tabular}{|c|c|c|c|c|c|}
\hline & \multicolumn{2}{|c|}{ Normal nutrition ( $n$ 125) } & \multicolumn{2}{|c|}{ Malnutrition ( $n$ 77) } & \multirow[b]{2}{*}{$P$ value } \\
\hline & Mean, $n$ or median & $\mathrm{SD}, \%$ or IQR & Mean, $n$ or median & $\mathrm{SD}, \%$ or IQR & \\
\hline $\begin{array}{l}\text { Age (years), mean and SD } \\
\text { Gender, } n \text { and } \%\end{array}$ & $59 \cdot 4$ & $15 \cdot 8$ & $64 \cdot 3$ & $16 \cdot 7$ & $\begin{array}{l}0.023^{*} \\
0.432^{N S}\end{array}$ \\
\hline Male & 72 & $57 \cdot 6$ & 40 & $51 \cdot 9$ & \\
\hline Female & 53 & 42.4 & 37 & $48 \cdot 1$ & \\
\hline BMI $\left(\mathrm{kg} / \mathrm{m}^{2}\right)$, median and IQR & $27 \cdot 5$ & $24 \cdot 5-30 \cdot 5$ & $21 \cdot 1$ & $19 \cdot 6-25 \cdot 5$ & $<0.001^{*}$ \\
\hline $\mathrm{BMl}<18.5 \mathrm{~kg} / \mathrm{m}^{2}, n$ and $\%$ & 0 & 0.0 & 10 & 12.9 & $<0.001^{*}$ \\
\hline $\mathrm{BMI} \geq 30.0 \mathrm{~kg} / \mathrm{m}^{2}, n$ and $\%$ & 91 & $72 \cdot 8$ & 73 & 94.8 & $<0.001^{*}$ \\
\hline Albumin $(g / l)$, median and IQR & 39.6 & $35 \cdot 2-43 \cdot 0$ & $37 \cdot 5$ & $33 \cdot 0-42 \cdot 0$ & $0.049^{*}$ \\
\hline Albumin $<32 \mathrm{~g} / \mathrm{l}, n$ and $\%$ & 19 & $15 \cdot 2$ & 16 & $20 \cdot 8$ & $0.228^{N S}$ \\
\hline
\end{tabular}

Normal nutrition, SGA classification A; malnutrition, SGA classification B and C; IQR, interquartile range; SGA, Subjective Global Assessment.

*Significant difference between SGA classification A and SGA classification B and C.

Table 2 Co-morbidity according to nutritional status among hospitalized non-surgical patients ( $n$ 202; median age 63.5 years) admitted to Nitra Teaching Hospital, Slovak Republic, April-June 2003

\begin{tabular}{|c|c|c|c|c|c|}
\hline \multirow[b]{2}{*}{ Co-morbidity } & \multicolumn{2}{|c|}{ Normal nutrition ( $n$ 125) } & \multicolumn{2}{|c|}{ Malnutrition $(n 77)$} & \multirow[b]{2}{*}{$P$ value } \\
\hline & $n$ & $\%$ & $n$ & $\%$ & \\
\hline CVD & 60 & 48 & 36 & 47 & NS \\
\hline Respiratory diseases & 19 & 15 & 14 & 18 & NS \\
\hline Neurological diseases & 18 & 14 & 5 & 6 & NS \\
\hline Endocrine diseases & 30 & 24 & 18 & 23 & NS \\
\hline Nephrologic diseases & 7 & 6 & 8 & 10 & NS \\
\hline Liver diseases & 13 & 10 & 12 & 16 & NS \\
\hline Gastrointestinal diseases & 10 & 8 & 19 & 25 & 0.001 \\
\hline Oncologic diseases & 31 & 25 & 31 & 40 & 0.021 \\
\hline Systemic diseases & 5 & 4 & 4 & 5 & NS \\
\hline Other diseases & 14 & 11 & 6 & 8 & NS \\
\hline
\end{tabular}

Normal nutrition, SGA classification A; malnutrition, SGA classification B and C; SGA, Subjective Global Assessment.

${ }^{*} P$ value for the difference between SGA classification A and SGA classification $B$ and $C$.

Table 3 Results of Cox regression models of 10-year survival among hospitalized non-surgical patients ( $n$ 202; median age 63.5 years) admitted to Nitra Teaching Hospital, Slovak Republic, April-June 2003

\begin{tabular}{lrccc}
\hline & $\beta$ & $\mathrm{HR}$ & $95 \% \mathrm{Cl}$ & $P$ value \\
\hline Model 1 without co-morbidity & & & & \\
Age & 0.002 & 1.00 & $1.00,1.00$ & $0.016^{*}$ \\
Male gender (ref. = female) & 0.374 & 1.45 & $0.97,2.18$ & 0.070 \\
BMl & 0.000 & 1.00 & $1.00,1.00$ & 0.720 \\
Albumin & -0.035 & 0.97 & $0.94 ; 0.99$ & $0.031^{*}$ \\
Malnutrition (ref. = normal & 0.440 & 1.55 & $1.04,2.32$ & $0.031^{*}$ \\
$\quad$ nutrition) & & & & \\
Model 2 with co-morbidity & & & & \\
Age & 0.039 & 1.04 & $1.02,1.06$ & $<0.001^{*}$ \\
Male gender (ref. = female) & 0.535 & 1.71 & $1.13,1.58$ & $0.011^{*}$ \\
BMl & -0.007 & 0.99 & $0.96,1.03$ & 0.745 \\
Albumin & -0.016 & 0.98 & $0.95,1.01$ & 0.298 \\
Malnutrition (ref. = normal & 0.231 & 1.26 & $0.80,1.99$ & 0.324 \\
nutrition) & & & & \\
Gastrointestinal disease & -0.639 & 0.53 & $0.25,1.11$ & 0.091 \\
Oncologic disease & 0.825 & 2.28 & $1.50,3.46$ & $<0.001^{*}$ \\
\hline
\end{tabular}

$\beta$, beta coefficient; HR, hazard ratio; ref., referent category; malnutrition, SGA classification B and C; normal nutrition, SGA classification A; SGA, Subjective Global Assessment.

*Significant predictor.

malnutrition was 6 (IQR 2-9) years and for patients with normal nutrition it was 9 (IQR 4-9) years. In-hospital mortality was low (six patients, $3.0 \%$ ), but during the following 10 years 105 patients died (52.0\%), forty-seven of whom were from the malnutrition group. A significantly higher proportion of patients with malnutrition died (61.0\%) compared with patients having normal nutrition (46.4\%; log-rank test $\chi^{2}=5 \cdot 37, P=0 \cdot 02$ ). Cox regression analysis controlling for age, gender, BMI and serum albumin showed that SGA was an independent predictor of death (hazard ratio $=1 \cdot 55 ; 95 \%$ CI $1 \cdot 04,2 \cdot 32, P=0 \cdot 031$; Table 3 and Fig. 1).

\section{Discussion}

Our study estimated the prevalence of malnutrition as assessed by SGA at $38.1 \%$. However, classification of malnutrition according to BMI was not sensitive enough; only the SGA A group had the same value of interpretation, as no patient with a BMI $<18.5 \mathrm{~kg} / \mathrm{m}^{2}$ was classified as A according SGA assessment. On the other hand, only $13.0 \%$ of malnourished patients according SGA classification B and $\mathrm{C}$ were assessed as undernourished using BMI. BMI assessment also estimated obesity (BMI $\geq 30 \cdot 0 \mathrm{~kg} / \mathrm{m}^{2}$ ) in four patients who were evaluated by SGA as undernourished. This is the reason why more sensitive and specific tools that 


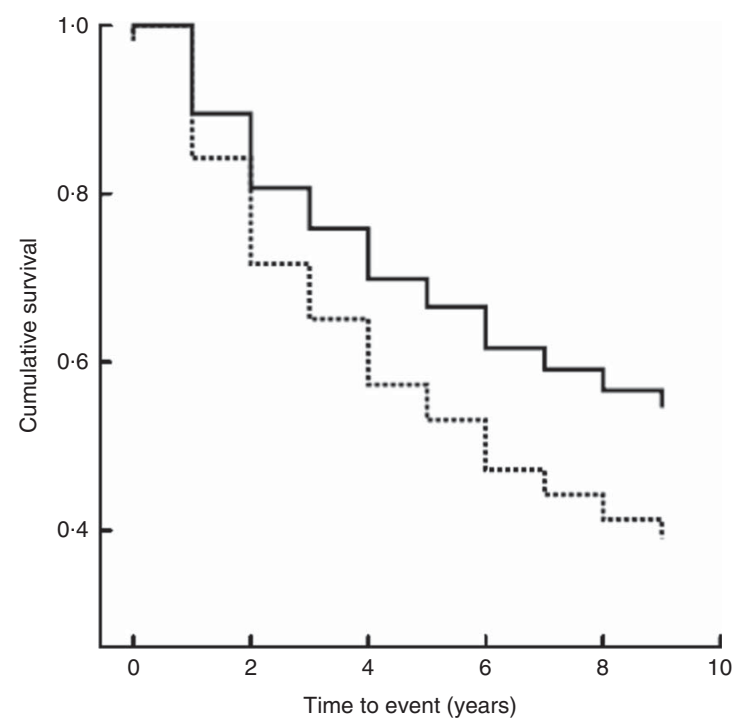

Fig. 1 Kaplan-Meier curves showing the association between nutritional status as evaluated by SGA (- normal nutrition, SGA classification $A ;-----$, malnutrition, SGA classification $B$ and $C$ ) and 10-year survival among hospitalized non-surgical patients ( $n$ 202; median age 63.5 years) admitted to Nitra Teaching Hospital, Slovak Republic, April-June 2003 (SGA, Subjective Global Assessment)

include weight loss as the key indicator for evaluating malnutrition must be used. The advisability of using BMI as an appropriate indicator of nutritional status has been widely discussed, especially in patients with heart failure ${ }^{(16)}$ or elderly residents of nursing homes ${ }^{(17)}$, and some recent studies have shown no associations between BMI and mortality risk in cancer or respiratory disease patients ${ }^{(6)}$. However, in some specific cases (nursing home residents), BMI showed superiority among three parameters considered to be nutritional predictors (BMI, Mini Nutritional Assessment score and serum albumin levels) ${ }^{(10)}$ and might be used as a simple screening tool, although its poor sensitivity must still be carefully considered.

Age is the most relevant confounding factor of malnutrition and we confirmed a higher prevalence of malnutrition assessed by SGA among older patients. Fewer men than women were malnourished according to SGA, but the differences between genders were not statistically significant.

Albumin is often advocated as being an indicator of malnutrition, but with the possible exception of kwashiorkor, serum albumin level is an unreliable marker of nutritional status $^{(18)}$. Other proteins as markers of malnutrition can also be estimated (transferrin, transthyretin, retinol-binding protein). There are many reasons (the half-life of albumin, the dependence of transferrin on Fe status, the rather expensive estimate of retinol-binding protein) why transthyretin is a very suitable marker for assessing efficacy in acute nutritional intervention. But for the clinical setting in Slovakia, albumin is the most common parameter of nutritional screening due to its general affordability. Individuals with simple malnutrition are rarely hypoalbuminaemic.
Serum albumin concentration does not often discriminate between well-nourished and malnourished patients ${ }^{(4)}$. In our cohort of patients hypoalbuminaemia was measured quite frequently in patients with good nutritional status (15.2\%), as well as in undernourished patients (20.8\%); the differences were not statistically significant. Therefore, in line with others, we consider serum albumin to be rather a marker of severity of concomitant illness rather than of nutritional status $^{(19)}$. Therefore, it is also reasonable to estimate plasma levels of inflammatory markers such as C-reactive protein, together with the proteins of nutritional status, in order to distinguish the inflammatory response of the body to nutritional status and malnutrition per se. Unfortunately, as our study was started 10 years ago, the routine measurement of C-reactive protein for general screening purposes without the presence of acute concomitant illness was not assigned as mandatory by health-care authorities. Nevertheless, hypoalbuminaemia in our study was found to be associated with mortality as an independent predictor of death; each decrease in albumin by $1.0 \mathrm{~g} / \mathrm{l}$ was associated with a $3 \%$ increase in mortality ${ }^{(20)}$. Similar data have been shown in studies of patients with end-stage renal disease ${ }^{(21)}$, after hip fracture $^{(22)}$ or in frail elderly patients ${ }^{(23)}$.

Malnutrition is commonly associated with certain diseases $^{(24,25)}$, so we explored the prevalence of malnutrition estimated by SGA among the co-morbidities. A statistically significant difference in the prevalence of malnutrition was clearly noted only in patients with gastrointestinal and oncologic diseases. Surprisingly, we failed to find a difference in the group of patients with liver diseases; this is probably due to low presence of end-stage liver disease in our sample. We have a similar explanation for the negative findings among patients with neurological diseases, probably due to the short time since onset of neurological disorders and the low number of patients with dementia in our cohort.

The impact of malnutrition on the higher need of hospitalization and increased in-hospital mortality was reported by Agarwal et al. ${ }^{(26)}$; weight loss of $15 \%$ or more of maximum body weight is also associated with increased risk of death ${ }^{(27)}$. Nutritional status also influences treatment tolerability in oncology patients ${ }^{(28)}$. Our study was underpowered to show any differences in short-term (in-hospital) mortality, as only six patients died, but we did find an association of malnutrition with long-term mortality at up to 10 years of follow-up. The impact of nutritional status on mortality was analysed using Cox regression models, controlled for age, gender, BMI and serum albumin level. Age and serum albumin level were independent predictors of survival, as expected. In addition, malnutrition as assessed by SGA was an independent predictor of death in a 10-year follow-up in the Slovakian health-care setting; the mortality of malnourished patients was $55 \%$ higher compared with patients with normal nutrition. Our findings are similar to results from studies in patients with various diseases ${ }^{(29,30)}$. However, when co-morbidity (oncologic and gastrointestinal) was added 
to our statistical analysis, nutritional status was no longer a significant predictor of survival; we expected a confounding effect from co-morbidity. The relatively small sample size might also have contributed to this result.

In summary, SGA is a simple screening tool of nutritional status and can be routinely used in Slovak hospitalized medical patients to indentify malnutrition, which predicts long-term risk of death. SGA may be used as a reliable and valid instrument for screening of malnutrition in patients who might benefit from some nutritional interventions; the effectiveness of such treatment should be prospectively evaluated.

\section{Acknowledgements}

Acknowledgements: The authors would like to thank the staff of the Department of Internal Medicine, Teaching Hospital Nitra (cooperating physicians, nurses, dietitians and administrative staff) for their kind support of this study. Financial support: This research received no specific grant from any funding agency in the public, commercial or not-for-profit sectors. The Slovak Society of Parenteral and Enteral Nutrition (SSPEV) initiated and supported the study (provided ethical support and advisory recommendations) with the aim of mapping the situation in one of Slovakia's largest hospitals and publishing the results of this study as the first data from Slovakia in this field. Conflict of interest: None. Authorship: V.K., design and coordination of the study, writing of the manuscript; J.R., design of the study and statistical analysis; M.G., collection of data; A.K., collection of data and statistical analysis. Ethics of buman subject participation: The study was approved by local ethics committee of the Nitra Teaching Hospital in Slovakia.

\section{References}

1. Gilmore SA, Robinson G, Posthauer ME et al. (1995) Clinical indicators associated with unintentional weight loss and pressure ulcers in elderly residents of nursing facilities. $J \mathrm{Am}$ Diet Assoc 95, 984-992.

2. McWhirter JP \& Pennington CR (1994) Incidence and recognition of malnutrition in hospital. BMJ 308, 945-948.

3. Charlton K, Nichols C, Bowden S et al. (2012) Poor clinical status of older subacute patients predicts clinical outcomes and mortality at 18 months of follow up. Eur J Clin Nutr $\mathbf{6 6}$, 1224-1228.

4. Addolorato G, Capristo E, Greco AV et al. (1998) Influence of chronic alcohol abuse on body weight and energy metabolism: is excess ethanol consumption a risk factor for obesity or malnutrition? J Intern Med 255, 387-395.

5. Numeroso F, Barilli AL \& Delsignore R (2008) Prevalence and significance of hypoalbuminemia in an internal medicine department. Eur J Intern Med 19, 587-591.

6. de Hollander EL, Van Zutphen M, Bogers RP et al. (2012) The impact of body mass index in old age on cause-specific mortality. J Nutr Health Aging 16, 100-106.

7. Detsky AS, Mc Langblin JR, Baber JP et al. (1987) What is subjective global assessment of nutritional status? JPEN J Parenter Enteral Nutr 11, 8-13.
8. Raslan M, Gonzalez MC, Torrinhas RS et al. (2011) Complementarity of Subjective Global Assessment (SGA) and Nutritional Risk Screening 2002 (NRS 2002) for predicting poor clinical outcomes in hospitalized patients. Clin Nutr 30, 49-53.

9. Vero LM, Byham-Gray L, Parrott JS et al. (2012) Use of the subjective global assessment to predict health-related quality of life in chronic kidney disease stage 5 patients on maintenance hemodialysis. J Ren Nutr 23, 141-147.

10. Veronese N, De Rui M, Toffanello ED et al. (2013) Body mass index as predictor of all-cause mortality in nursing home residents during a 5-year follow up. J Am Med Dir Assoc 14, 53-57.

11. Pirlich M, Schutz T, Norman K et al. (2006) The German hospital malnutrition study. Clin Nutr 25, 563-572.

12. Schindler K, Pernicka E, Laviano A et al.; NutritionDay Audit Team (2010) How nutritional risk is assessed and managed in European hospitals: a survey of 21,007 patients findings from the 2007-2008 cross-sectional nutritionDay survey. Clin Nutr 29, 552-559.

13. Vanderwee K, Clays E, Bocquaert I et al. (2010) Malnutrition and associated factors in elderly hospital patients: a Belgian cross-sectional, multi-centre study. Clin Nutr 29, 469-476.

14. World Health Organization (2003) Global Health Observatory (GHO). Mean Body Mass Index (BMI). Situation and trends. http://www.who.int/gho/ncd/risk_factors/bmi_text/ en/ (accessed March 2012).

15. Garrow JS (1998) Three limitations of the body mass index. Am J Clin Nutr 47, 553.

16. Gastelurrutia P, Lupon J, Domingo M et al. (2011) Usefulness of body mass index to characterize nutritional status in patients with heart failure. Am $J$ Cardiol 108, 1166-1170.

17. Kimyagarov S, Klid R, Levenkrohn S et al. (2010) Body mass index (BMI), body composition and mortality of nursing home elderly residents. Arch Gerontol Geriatr 51, $227-230$

18. Friedman AN \& Fadem SZ (2010) Reassessment of albumin as a nutritional marker in kidney disease. J Am Soc Nephrol 21, 223-230.

19. Zadak Z (2002) Interpretace plazmatických hladin bílkovin a hodnocení viscerálního protein. In Vyživa $v$ intenzivní péči (Publication 1555), 1st edn., pp. 74-95. Praha: Grada Publishing, a.s.

20. Hrnciarikova D, Juraskova B, Hyspler R et al. (2007) A changed view of serum prealbumin in the elderly: prealbumin values influenced by concomitant inflammation. Biomed Pap Med Fac Univ Palacky Olomouc Czech Repub 151, 273-276.

21. Dashti N, Einollahi N, Nabachtian F et al. (2012) Significance of albumin and C-reactive protein variations in 300 end stage renal disease patients in Teheran University of Medical Science Hospitals during year 2010. Acta Med Iran 50, 197-202.

22. Miyanishi K, Jingushi S \& Torisu T (2010) Mortality after hip fracture in Japan: the role of nutritional status. J Orthop Surg (Hong Kong) 18, 265-270.

23. Kitamura K, Nakamura K, Nishiwaki T et al. (2010) Low body mass index and low serum albumin are predictive factors for short- term mortality in elderly Japanese requiring home care. Tohoku J Exp Med 221, 29-34.

24. Davies SJ, Philips L, Naish PF et al. (2002) Quantifying comorbidity in peritoneal dialysis patients and its relationship to other predictors of survival. Nephrol Dial Transplant 17, 1085-1092.

25. Charlson ME, Pompei P, Ales Kl et al. (1987) A new method of classifying prognostic comorbidity in longitudinal studies: development and validation. J Chronic Dis 40, 373-383.

26. Agarwal E, Ferguson M, Banks M et al. (2013) Malnutrition and poor food intake are associated with prolonged hospital 
stay, frequent readmissions, and greater in-hospital mortality: results from the Nutrition Care Day Survey 2010. Clin Nutr 32, 737-745.

27. Ingram DD \& Mussolino ME (2010) Weight loss from maximum body weight and mortality: the Third National Health and Nutrition Examination Survey linked mortality file. Int J Obes (Lond) 34, 1044-1050.

28. Barret M, Malka D, Aparicio T et al. (2011) Nutritional status affect tolerability and survival in metastatic colorectal cancer patients: results of an AGEO prospective multicenter study. Oncology 81, 395-402.

29. Rodriguez-Pecci MS, Carlson D, Montero-Tinnirello J et al. (2010) Nutritional status and mortality in community acquired pneumonia. Medicina (B Aires) 70, 120-126.

30. Castellanos Fernandez M, Santana Porben S, García Jordá E et al. (2008) Influence of hyponutrition on occurrence of complications and mortality among cirrhosis patients. Nutr Hosp 23, 68-74. 